\title{
Research on the Service Quality of the Last-Mile Logistics Distribution
}

\author{
Yan Li", Sumei Li \\ School of Management, China University of Mining and Technology (Beijing), Beijing, China \\ Email address: \\ liyan@cumtb.edu.cn (Yan Li) \\ ${ }^{*}$ Corresponding author
}

\section{To cite this article:}

Yan Li, Sumei Li. Research on the Service Quality of the Last-Mile Logistics Distribution. Science Innovation. Vol. 6, No. 3, 2018 , pp. 156-163. doi: 10.11648/j.si.20180603.17

Received: April 10, 2018; Accepted: May 25, 2018; Published: June 22, 2018

\begin{abstract}
B2C e-commerce has put forward higher requirements on the ability of logistics distribution, in which the last-mile delivery quality plays a significant role. Recently, the introduction of intelligent terminals generates a new last-mile delivery mode. Centered with the service quality of this new mode, this paper firstly analyzes the features of the new last-mile delivery mode with intelligent terminals as the carrier, then identifies the dimensions of service quality in this context, and thirdly analyzes the relationships among features of the new last-mile delivery mode, service quality, trust in the service provider, and customer satisfaction toward the last-mile distribution service. A structrual equation model is run on the data collected through survey. It concludes that richness of interactive media and convenience of self-service can significantly improve the sevice quality of the last-mile delivery, and through the mediating role of trust, quality service promotes customer satisfaction indirectly.
\end{abstract}

Keywords: Last- Mile Distribution, Service Quality, Trust, Customer Satisfaction

\section{最后一公里物流配送的服务质量研究}

\section{李岩”, 李素梅}

管理学院, 中国矿业大学 (北京), 北京, 中国

\section{邮箱}

liyan $a$ cumtb.edu.cn (李岩)

摘要：以B2C为代表的电商行业网购业务发展迅速，对物流行业的配送能力提出了更高要求，尤其是最后一公里配送 质量关系到网络购物成败。智能化终端的引入，产生了一种新型的最后一公里配送模式，本文针对该新型配送模式的 服务质量展开研究, 以识别能够提升该新型模式服务质量的路径。本文首先分析以智能化终端为载体的最后一公里新 型配送模式的特点, 然后明确在这种新型配送模式下服务质量内涵, 并提出这种新型配送模式服务质量的影响因素以 及服务质量对信任和顾客满意度的影响作用，最后通过问卷调研的形式收集数据并进行假设检验。本文得出如下结论: 交互媒介的丰富性和自助服务的便利性能够显著提升最后一公里配送的服务质量, 并且服务质量通过信任间接提升顾 客满意度。

关键词: 最后一公里配送, 服务质量, 信任, 顾客满意度 


\section{1. 引言}

物流过程包括仓储、装卸、流通加工、运输、末端配 送, 其中末端配送也被称为最后一公里配送, 服务提供方 主要在这一阶段与顾客交互, 因此对物流服务提供方来说 十分重要。然而在此阶段中由于服务人员及顾客的不确定 性, 使得此环节出现的问题颇多, 例如, 单一化的配送业 务及顾客个性化的收货时间, 导致服务提供方的工作人员 工作积极性和效率降低, 且时效性差的配送服务使得终端 顾客体验差且对特定服务提供方的依赖程度降低, 从而引 发顾客对物流服务提供方的信任缺失, 加剧双方之间的矛 盾。中国电子商务投诉平台发布的数据表明, 在过去一年 的B2C业务投诉中, 物流末端配送环节的投诉占比近 $10 \%$, 且呈现不断上升趋势。

最后一公里配送的重要性吸引学者们从多个视角研 究如何提升最后一公里的配送服务。一部分研究从技术层 面考察了最后一公里配送的优化问题, 例如自提点布局规 划、配送路线优化、运输工具选择等。一部分研究从供应 链角度分析了供应链各个主体之间的博弯对最后一公里 配送服务的影响 [1]。由于最终消费者是最后一公里配送服 务的重要参与者, 因此还有部分研究从如何提升服务质量 的角度研究最后一公里配送服务, 例如杨晓红和严中华基 于SERVPERF研究了最后一公里配送服务的质量[2]。

随着互联网新兴信息技术的发展, 在最后一公里这一 关键环节中, 对应的配送模式越来越智能化, 其中智能化 终端作为最后一公里配送核心的模式最为典型。智能化终 端是一种基于互联网技术集成快递投递和提取的自助服 务设备[3]。本文将这种最后一公里的配送模式称为中心辐 射配送模式。由于智能化终端鲜明的技术特征, 现有研究 主要研究了智能化终端的技术特性。例如, 一些研究计算 得出智能化终端的出现降低了快递配送距离, 降低了二氧 化碳的排放量, 提升了快递配送的包裹数量 [4]。一些研究 从智能化终端的设施布局、智能终端设备的数量、空间大 小等方面优化了智能化终端的配置, 最大化智能化终端运 营商的收益[5]。借助智能化终端, 顾客可以自由和灵活的 参与到最后一公里物流配送环节, 因此, 顾客价值的提升 是智能化终端这种模式不可缺少的一部分, 最近一些研究 开始从这个角度分析智能化终端的价值 [6]。

智能化终端的技术特征会最终体现在顾客对这种配 送模式的服务感知之中。但是, 目前鲜有将二者联系起来 研究智能化终端的配送问题。因此, 本文的研究目的是探 讨在中心辐射模式下如何提升最后一公里物流配送服务 质量。

\section{2. 理论背景}

\section{1. 中心辐射模式特点}

中心辐射模式依靠智能化的基础设施, 包括后台数据 系统和自助式机器设备等, 使得此模式拥有传统模式不具 有的更加丰富的交互媒介和便利的自助服务。
交互媒介的丰富性是指，顾客能运用十分丰富化的交 互工具参与物流配送过程, 这使得顾客在参与活动的过程 中获得极佳的服务质量体验。中心辐射模式的信息化将以 往的人-人双向交互的强交互模式转化为便捷的单向的人机交互模式，实现了人与人关系的交互自由化，从而可以 提高服务提供方的工作人员服务效率，与此同时也提升顾 客感知到的服务质量。例如, 通过微信、第三方信息服务 类APP等、电话、短信等信息平台等多种手段可以使得服 务更加优质和高效, 顾客体验更加自由化和友好化。而在 传统模式下，只存在人-人交互方式，交互媒介单调，易 造成服务质量的下降。

自助服务的便利性, 是指顾客能自由的安排业务办理 时间且能通过自己一方的能力完成业务的可能程度, 在这 个过程中顾客是借助智能化技术完成业务办理。由于互联 网技术的持续革新, 使得自助服务方式逐渐的普及化, 应 用范围越来越大。例如, 借助于密码取件信息系统, 顾客 在自提柜界面输入特定密码信息后, 就能随时随地顺利取 件, 不受服务提供方营业时间限制, 这种 24 小时式自助服 务模式, 明显地减少了顾客的时间和精力投入。当在取件 过程中遭遇障碍时, 均能轻易传递到相应的信息平台, 服 务提供方的工作人员实时监控并及时处理, 减少了传统快 递模式中的服务响应时间。下表总结了中心辐射模式的特 点。

表1 中心辐射模式两个特点的内涵。

\begin{tabular}{ll}
\hline 特点 & 内涵 \\
\hline & 顾客参与过程中所能使用的媒介交流工具比较丰富, \\
& 有许多可以自由选择的活动参与方式。 \\
交互媒介的 & 借助于丰富化的媒介工具, 相应的工作人员单位工作 \\
丰富性 & 内容耗时下降, 工作效率大大提升。 \\
& 基于数据系统高度智能信息化, 交互媒介的丰富性才 \\
& 得到充分体现, 是顾客和服务提供者双方都受益。 \\
& 由于存在自助服务功能, 服提供方的工作人员能够很 \\
& 灵活地安排工作内容, 服务便利化效应明显。 \\
& 自助服务的自助服务功能, 所有的顾客能够自由的安排自己 \\
便利性 & 取件的时间, 即顾客取件时间安排自主性提高。 \\
& 强大自助功能下, 顾客能感受到极大服务便利性, 使 \\
& 得顾客抱怨率较低。 \\
\hline
\end{tabular}

\section{2. 服务质量}

服务质量是顾客对特定服务内容体验的综合评价[7]。 先前研究经过大量测试归纳出服务质量的SERVQUAL 模 型, 认为服务质量由五个维度构成: 有形性、可靠性、保 障性、移情性、响应性 $[8,9]$ 。根据该模型, 本文提出了 中心服务模式服务质量的具体内涵，见表2所示:

表2 中心服务模式服务质量内涵。

\begin{tabular}{lll}
\hline 维度 & 含义 & 具体解释 \\
\hline & & 智能化的设备 \\
有形性 & 指员工的衣着和运 & 服务设施具有吸引力 \\
& 行的机器设备 & 员工能提供专业的服务 \\
& & 服务硬件吻合提供的服务内容 \\
& 指借助人工设施兑 & 按规定的要求完成服务内容 \\
可靠性 & 有能力协助顾客破除障碍 \\
& 服务承诺的能力 & 公司是值得依靠的 \\
\hline
\end{tabular}




\begin{tabular}{|c|c|c|}
\hline 维度 & 含义 & 具体解释 \\
\hline \multirow{5}{*}{ 保障性 } & \multirow{5}{*}{$\begin{array}{l}\text { 指服务人员的工作 } \\
\text { 素养较高 }\end{array}$} & $\begin{array}{l}\text { 能准确无误的提供服务 } \\
\text { 能正确告知顾客服务内容 }\end{array}$ \\
\hline & & 服务人员值得信赖 \\
\hline & & 顾客对服务内容十分信任 \\
\hline & & 员工十分有素养 \\
\hline & & 员工通过进修后提高服务能力 \\
\hline \multirow{3}{*}{ 移情性 } & \multirow{3}{*}{$\begin{array}{l}\text { 指用心对待顾客并 } \\
\text { 为其提供服务 }\end{array}$} & $\begin{array}{l}\text { 为不同的顾客提供不同的服务 } \\
\text { 为顾客提供个性化的关心 }\end{array}$ \\
\hline & & 员工了解关注顾客所有要求 \\
\hline & & 开放的时间能照顾全部顾客 \\
\hline \multirow{3}{*}{ 响应性 } & \multirow{3}{*}{ 指能及时地帮助顾 } & $\begin{array}{l}\text { 顾客能掌握公布的时间 } \\
\text { 能及时提供服务 }\end{array}$ \\
\hline & & 员工即使忙碌时也愿意给予帮助 \\
\hline & & 服务人员以较快的速度提供帮助 \\
\hline
\end{tabular}

\section{3. 服务质量与信任、顾客满意度的关系}

针对传统渠道服务的研究发现服务质量和顾客满意 度之间存在着关联性, 即服务质量水平越高, 对于的顾客
满意度水平也越高 $[10,11]$ 。而在当今线上系统服务中, 信任成为了一个重要变量。信任是服务提供方对顾客的服 务能力担保和承诺, 是双方通过若干次交互后形成彼此依 赖的信任关系 $[12,13]$ 。在移动互联网高度发展的现在, 尤其是大数据技术的日益成熟，各类公共及个人的信息极 容易被暴露, 使得顾客对系统的使用产生疑虑。若能让顾 客让顾客对这些系统及背后的服务商产生信任, 就能够提 升顾客对服务的满意程度[14]。而服务质量的下降则会影 响顾客对服务的信任 [15]。因此, 本文提出在中心辐射模 式下的最后一公里服务中, 信任会在服务质量和顾客满意 度之间产生不可忽视的中介作用。

\section{3. 研究模型}

根据前述理论背景, 本文构建了如下图所示的中心辐 射模式下最后一公里服务质量模型。

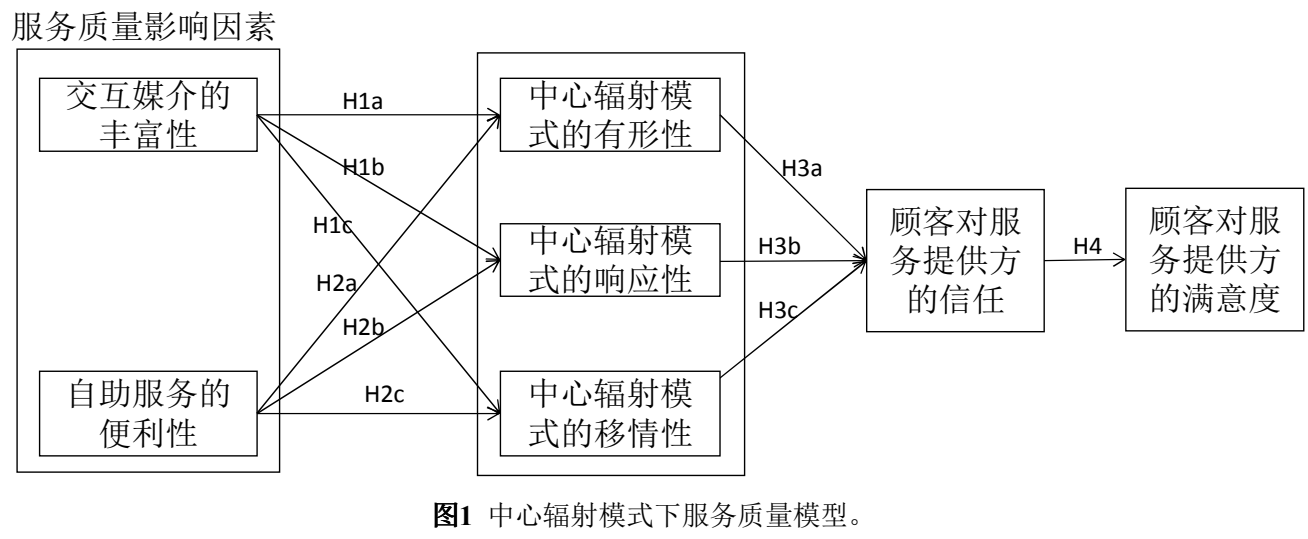

\section{1. 交互媒介的丰富性与服务质量的关系}

交互媒介的丰富性是指信息沟通工具或平台的丰富 性, 信息受众能通过丰富化的媒介工具满足其个性化需求, 且交互方式简单分为人-机交互和人-人交互[16]。针对中 心辐射模式的物流配送, 本文将交互媒介的丰富性定义为: 顾客能使用多种现代智能化的媒介工具和平台参与整个 物流配送服务过程。通过例如微信、短信、QQ等社交媒 介工具, 使得最后一公里配送过程的交互媒介方式呈现出 极强的丰富性。在中心辐射模式中, 交互媒介的丰富性主 要体现在顾客能通过面对面、移动互联网或电话等方式参 与到物流配送服务中, 并且能通过Led显示屏、宣传海报、 二维码等丰富化形式实现人 (服务提供方) -人 (顾客人) 交互、人 (顾客) - 机 (智能化设备设施) 交互等多种交 互方式[17]。本文提出中心辐射配送模式的交互媒介的丰 富性越强, 越能够提升顾客所感知到的配送服务质量。

第一, 交互媒介的丰富程度能够提升顾客感知到的物 流配送服务的有形性。也就是说, 服务提供方在与顾客互 动过程中, 会借助多种多样的媒介工具让顾客体验到良好 的服务质量, 而这些媒介工具必须配套的服务设备来支持, 以使得各类交互过程正常顺利的开展。以近邻宝为例来说, 在智能化的信息系统后台背景下, 顾客主要通过特定短信 信息码、二维码等完成取件等业务办理活动, 并且顾客在
参与过程中能很直接、直观的感知近邻宝提供的智能化服 务设施, 这些直观且看得见的服务设施和相应的工作人员 使顾客感到安全和放心, 不会担心近邻宝是否有能力提供 对应的服务, 从而顾客对其服务质量的体验和评价也会较 高, 也就是交互媒介的丰富性程度越强, 对应顾客感受到 的服务质量中的有形性程度也会越强。

第二, 交互媒介的丰富程度能够提升物流配送服务对 顾客需求的响应性。在中心辐射模式中, 通过多种交互方 式, 中心辐射模式的物流配送服务方可以即时告知顾客准 确的服务时间。即使当现场工作人员忙碌时, 也能通过其 他的交互方式及时为顾客提供所需的服务。以高校校园中 的近邻宝来说, 由于服务对象主要是老师和学生, 这个群 体因为上课下课的时间原因, 不能随时随地完成日常取件 寄件业务, 但由于上下课时间是比较固定的, 近邻宝后台 信息系统可以记录顾客的业务参与信息, 形成一份份个性 化的信息档案, 可以在下课时段高峰期配备充足的服务人 员, 在上课或周末等相对低谷时间段减少人员配备, 因此, 服务提供方能以合适的时间及合适的人员来满足顾客需 要十分及时且准确的服务需求, 也就是, 交互媒介的丰富 性能大大提高服务质量中的响应性程度 [18]。

第三, 交互媒介的丰富程度还能够增强顾客感知到的 物流配送服务的移情性。在中心辐射模式中，服务提供方 
所具备的丰富化交互媒介能使顾客感受到个性化的服务。 在最后一公里配送的社区服务中，通过手机短信、飞信、 微信、客户端等多种媒介方式, 来通知顾客的快件是否到 达以及具体存放位置。由于许多顾客是日常行程比较繁忙 的上班族, 为了防止顾客遗忘取件或者减少顾客等待服务 的时间, 设置有多次短信提醒, 并且顾客可以根据自身的 需求选择自助下单还是人工下单。丰富化的交互媒介工具 能够让顾客感受到其服务需求和个人利益得到照顾, 这样 针对不同顾客群的个性化服务, 能够极大增加顾客对服务 质量中移情性的感受程度。

因此, 本文提出以下假设:

H1a: 交互媒介的丰富性越强, 越能够提升顾客感受 到的物流配送服务的有形性;

H1b: 交互媒介的丰富性越强, 越能够提升物流配送 服务对顾客需求的响应性;

H1c：交互媒介的丰富性越强, 越能够增强顾客感知 到的物流配送服务所具有的移情性。

\section{2. 自助服务的便利性与服务质量的关系}

自助服务的便利性指在智能化背景下借助交互性终 端设备、互联网连接等方式, 顾客不需要与工作人员面对 面交接和互动, 就能满足其服务需求 [19]。针对中心辐射 模式的物流配送，本文对自助服务的便利性的定义是：在 最后一公里背景下的中心辐射中, 顾客能自行通过信息化 的自助服务设施或设备来满足服务需求的便利性程度。在 中心辐射模式中, 自助服务的便利性主要体现自助服务设 备使用起来十分容易, 所列出的操作提示流程十分清晰明 确, 顾客不需要具备有关自助取件服务的专业知识, 就能 通过很少的操作环节自助完成全部过程。本文提出中心辐 射配送模式的自助服务的便利性越强, 越能够提升顾客所 感知到的配送服务质量。

第一, 自助服务的便利程度能够提升顾客感知到的物 流配送服务的有形性。在中心辐射模式的物流配送活动中, 自助服务的实现需要借助若干固定的设施设备来支持服 务实现, 另外顾客在自助服务过程中所感受的便利性程度 受配套的自助服务设施智能程度的影响。在中心辐射模式 的配送服务中, 当顾客进行寄件业务时, 只需要向近邻宝 服务人员口头告知姓名、手机号、寄送地址等信息，通过 近邻宝的自提式终端填写设备, 就能快速打印出详细准确 的寄件面单信息, 操作方便的自助终端业务设备, 能让顾 客需求的服务顺利完成。并且由于这种一一对应的服务需 求信息, 不会让服务提供方在为顾客服务的整个环节中出 现任何信息方面的失误。即自助服务的便利性程度越强, 顾客在参与配送服务活动时, 对其服务质量中的有形性维 度感受越强[20]。

第二, 自助服务的便利程度能够提升顾客感知到的物 流配送服务的响应性。在中心辐射模式中, 基于操作简易 的自助服务设备, 顾客能根据自己实际时间安排来享受及 时高效的服务。以社区近邻宝为例, 当家里的年轻人都处 于繁忙的上班状态时, 家里大多只剩下老人时, 如果年轻 上班族网购的家居用品到达小区近邻宝但无法亲自取件 时 [21], 由于近邻宝简易的操作流程, 不具备相关自助服
务设备知识的老人也能及时准确的完成取件, 即使在取件 过程中遇到障碍, 相应的工作人员也会提供及时有效的帮 助满足所有顾客的需求 [22]。因此, 无论是何种年龄阶段、 何种职业身份的顾客, 即使他们不具备丰富的自助设备操 作等专业知识, 也能通过便利性极强的自助服务终端或工 作人员的共同帮助, 来体验到及时且准确的配送服务。综 上所述, 助服务的便利性越高顾客所感受到的服务质量中 响应性程度越明显。

第三, 自助服务的便利程度能够提升顾客感知到的物 流配送服务的移情性。在中心辐射模式的智能信息化背景 下, 通过智能化设备连接, 能使顾客享受到个性化服务且 顾客的个人利益（譬如个人隐私）能得到很好的保护。在 高校校园的近邻宝中, 一般老师和学生总数较大, 不能保 证所有的顾客够已经下载了近邻宝客户端, 对于没有下载 其客户端且需要快速满足服务需求的顾客, 由于操作简易 的自助服务设备, 近邻宝工作人员可以简单演示后由顾客 实现自己个性化的服务需求, 并且由于一对一独立的自助 操作环境, 顾客的个人信息不会被泄露出去, 而是会通过 自助服务大数据平台后, 根据顾客多次的操作痕迹来更加 精准化为顾客下次的服务需求做准备[23]。因此, 通过自 助服务的便利性, 顾客的个性化需求得到满足, 且顾客个 人利益及个人信息都得到很好的保护, 也就是自助服务的 便利性越明显, 顾客感受到的物流配送服务质量中的移情 性程度越高。

因此, 本文提出以下假设:

H2a：自助服务的便利性越明显, 越能够提升顾客感 受到的物流配送服务的有形性;

$\mathrm{H} 2 \mathrm{~b}$ ：自助服务的便利性越明显，越能够提升物流配 送服务对顾客需求的响应性;

$\mathrm{H} 2 \mathrm{c}$ : 自助服务的便利性越明显, 越能够增强顾客感 知到的物流配送服务所具有的移情性。

\section{3. 服务质量与信任的关系}

信任是由能力、善意、一致性这三个维度组成, 即为 一方有能力提供且及时提供所承诺的服务, 其结果是一方 对另一方形成极强的信赖感 [24]。针对中心辐射模式的物 流配送, 本文将信任定义为: 在服务提供方多次顺利完成 向顾客承诺的服务内容后, 会得到顾客对其服务能力的肯 定，与此同时顾客因为多次优质的服务体验从而对其提供 的服务感到有信心。在中心辐射模式中, 信任主要体现在 顾客对工作人员专业素养的信赖和对特定服务提供方的 放心程度。

本文提出中心辐射配送模式的服务质量越高, 顾客对 其服务的信任程度越强。

第一，服务质量中的有形性越明显，顾客对其物流配 送服务的信任程度越强。在中心辐射模式中, 物流服务方 能通过看得见的服务设施来为所有顾客提供服务, 由于此 模式下的服务设施智能信息程度较高, 顾客在参与过程中 会感到放心。以近邻宝为例, 当顾客进行普通物品邮寄时, 可以通过真实存在的顾客服务台或自助机器设备来办理 相应业务, 并且当物品因意外情况发生损坏 (尤其是贵重 物品）顾客可以依据面对面填写的面单来协商理赔细节, 
不用担心物品丢失或者理赔时找不到相应的理赔对象, 这 使得顾客对近邻宝感到很信赖[25], 也就是服务质量中的 有形性越明显, 顾客对物流配送服务提供方的信任程度越 强。

第二, 服务质量中的响应性越明显, 顾客对其物流配 送服务能力的信任程度越强。在中心辐射模式中, 服务提 供方的工作人员会准确告诉顾客能提供服务的时间, 并且 工作人员在忙碌时也能为顾客提供及时的服务, 这极大的 增强了顾客对工作人员和该服务的信任度。在商务写字楼 配送服务中, 由于办公区域或线上平台会告知顾客能提供 服务的时间，顾客通过线上查询后会合理安排时间，这大 大减少了顾客因工作人员提前下班或者因事离开而浪费 的时间。并且当顾客有紧急业务要办理时, 譬如商务上的 往来函件寄送, 工作人员即使再忙碌也会帮顾客顺利办理, 这使得顾客对其提供的服务十分信赖, 也就是服务提供方 较强的配送能力，使得顾客对其服务的信任提升。

第三，服务质量中的移情性越明显，顾客对其物流配 送服务的信任程度越强。因为服务提供方能够为不同顾客 提供个性化的服务, 当发生意外情况是也能满足顾客需求。 譬如在社区近邻宝中, 当顾客在工作日购买的郊游用品需 在周末才能到达, 顾客只需要提前在网上下单时预约异地 取件时间段和具体地址, 就能在周末异地郊游地也能顺利 取件以满足郊游所需, 顾客个性化的需求和利益得到满足 后, 其对近邻宝提供的服务会感到十分信任[26], 也就是 服务质量中的移情性体现了物流配送方照顾到顾客的利 益, 因而提升了信任。

因此, 本文提出以下假设:

$\mathrm{H} 3 \mathrm{a}$ ：服务质量中的有形性越明显, 越能够提升顾客 对特定物流配送服务的信任程度;

$\mathrm{H} 3 \mathrm{~b}$ ：服务质量中的响应性越明显, 越能够提升顾客 对特定物流配送服务的信任程度;

$\mathrm{H} 3 \mathrm{c}$ : 服务质量中的移情性越明显, 越能够提升顾客 对特定物流配送服务的信任程度;

\section{4. 信任与顾客满意度的关系}

Cardozo认为顾客满意度是指顾客对期望的需求是否 被满足的感受程度和评价结果。针对中心辐射模式的物流 配送, 本文将顾客满意度定义为: 在中心辐射模式下, 顾 客对自己选择的服务提供方所提供服务感到满意的程度 [27]。本文中顾客满意度体现在, 顾客认为自己选择的服 务提供方和参与方式是十分正确且明智的, 不会对自己的 选择感到后悔或不满意[28]。

本文提出顾客对服务提供方的信任程度越强, 越能够 提升顾客对其配送服务的满意程度。

在中心模式中, 可以把服务提供方提供的服务活动分 为活动前、活动中及活动后这三个细分阶段。顾客通过这 三个阶段的服务预期和实际服务体验的差距来形成对其 服务提供方提供的服务的信任程度, 从而去影响到最终的 顾客满意度。在实际服务过程参与中, 比如信息共享、双 方合作关系的投入等会有助于顾客信任的形成及增强, 会 使顾客认为自己的选择是明智且正确的, 会为自己的选择 感到高兴和满意, 从而达到极好的顾客满意度水平[29]。
以近邻宝为例, 当顾客对邮寄物品的物流速度和时效性要 求较高时, 会仔细对比与近邻宝类似的服务方之后, 选择 使用近邻宝满足其服务需求并包含对其较高的服务预期。 在与近邻宝工作人员的业务服务需求交流过程中, 其信息 后台系统、客户端APP平台等会让顾客实时信息共享，使 顾客对其信任程度的加深, 尤其当物品如顾客期望完成后, 会让顾客觉得选择近邻宝这项服务是正确的且令人满意 的 [30], 即近邻宝分别从能力 (有能力满足顾客的服务需 求）、善意（顾客的利益会得到保障）、一致性（顾客的 服务期望和实际服务体验一致）信任的维度来提高顾客 对其提供的服务的满意度。因此顾客对特定对象的信任程 度越强, 会使得其在参与该项服务过程中的满意度越高。

因此, 本文提出以下假设:

H4: 顾客对物流配送服务的信任程度越强, 越能够提 升顾客的满意程度。

\section{4. 实证分析}

\section{1. 数据收集}

本论文利用likert五级量表测量每个变量, 设计调研问 卷收集数据。测量指标选用现有文献中所使用的量表, 并 根据研究问题进行了相应的修订。问卷发放采用随机抽样 的方式, 针对使用网络购物的消费者群体。发放范围包括 包含6所高校以及 4 个街道社区, 共发放了 408份问卷, 实 际收回的数量为386份,其中有效问卷332份, 问卷实际回 收率为 $81.4 \%$ 。样本涵盖年龄在 20 岁 40 岁之间的人群, 超 过 $54 \%$ 的样本每月进行 3 次及以上的网购。

\section{2. 量表检验}

表3所示了本文量表的信度检验结果, 每个变量的 Composite reliability都大于 0.7 , 即满足内部一致性的基本 标准要求[31]。

表3 变量信度表。

\begin{tabular}{|c|c|c|c|c|}
\hline 变量名称 & 指标数 & $\begin{array}{l}\text { Composite } \\
\text { reliability }\end{array}$ & 因子载荷 & 交叉载荷 \\
\hline 有形性 & 3 & 0.874 & $0.807 \sim 0.859$ & $0.295 \sim 0.576$ \\
\hline 响应性 & 4 & 0.888 & $0.773 \sim 0.866$ & $0.368 \sim 0.645$ \\
\hline 移情性 & 3 & 0.891 & $0.823 \sim 0.887$ & $0.236 \sim 0.679$ \\
\hline $\begin{array}{l}\text { 交互媒介的丰 } \\
\text { 富性 }\end{array}$ & 4 & 0.896 & $0.734 \sim 0.870$ & $0.315 \sim 0.565$ \\
\hline $\begin{array}{l}\text { 自助服务的便 } \\
\text { 利性 }\end{array}$ & 3 & 0.836 & $0.781 \sim 0.813$ & $0.295 \sim 0.519$ \\
\hline $\begin{array}{l}\text { 顾客对服务提 } \\
\text { 供方的信任 }\end{array}$ & 6 & 0.934 & $0.756 \sim 0.873$ & $0.434 \sim 0.664$ \\
\hline $\begin{array}{l}\text { 顾客对服务提 } \\
\text { 供方的满意度 }\end{array}$ & 5 & 0.947 & $0.840 \sim 0.910$ & $0.346 \sim 0.685$ \\
\hline
\end{tabular}

依据表 3 中的结果显示, 每个测量指标在所属潜变量 上的因子载荷值大于其他测量指标在该潜变量上的因子 载荷值, 也大于该测量指标在其他潜变量上的因子载荷值。 同时, 如 
表4所示，各个潜变量之间的相关系数均小于每个潜 变量AVE值的平方根。这些检验说明模型的潜变量之间具
有较好的区别效度。此外, 每个潜变量的AVE值都大于 0.5 , 表明量表具有较高的聚敛效度。

表4 变量相关系数及AVE。

\begin{tabular}{|c|c|c|c|c|c|c|c|c|}
\hline & AVE & SFS & $\overline{\text { EPT }}$ & $\overline{R S P}$ & TGB & TRS & MDA & STF \\
\hline SFS & 0.630 & 0.827 & & & & & & \\
\hline EPT & 0.732 & 0.379 & 0.855 & & & & & \\
\hline RSP & 0.664 & 0.499 & 0.699 & 0.815 & & & & \\
\hline TGB & 0.698 & 0.619 & 0.501 & 0.625 & 0.835 & & & \\
\hline TRS & 0.704 & 0.586 & 0.632 & 0.670 & 0.582 & 0.839 & & \\
\hline MDA & 0.685 & 0.491 & 0.560 & 0.507 & 0.457 & 0.563 & 0.794 & \\
\hline STF & 0.783 & 0.490 & 0.485 & 0.540 & 0.471 & 0.732 & 0.468 & 0.885 \\
\hline
\end{tabular}

注：TGB (有形性)，RSP (响应性)，Empathy（移情性），SFS（自助服务的便利性），MDA（交互媒介的丰富性）， TRS（信任），STF（满 意度）。对角线为每个潜变量AVE值的平方根。

\section{3. 模型路径系数分析}

本文利用smartp1s2.0软件来构建结构方程模型, 并进行求解, 结果如图2所示:

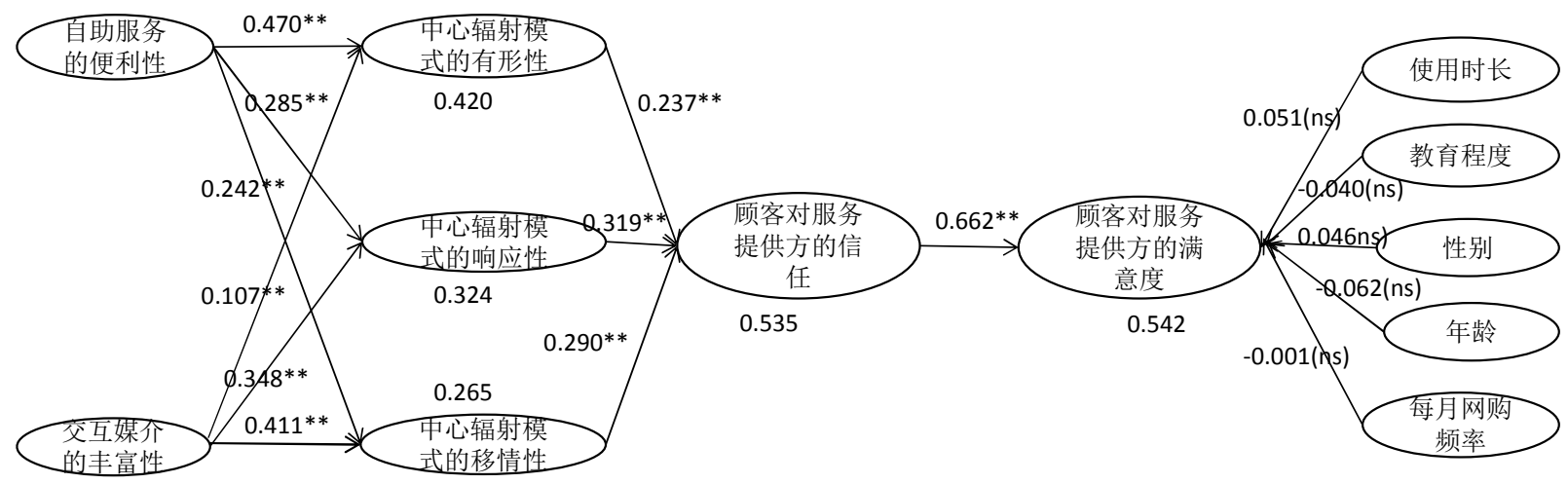

图2 结构分析结果。

注: $+\mathrm{p}<0.1, * \mathrm{p}<0.05, * * \mathrm{p}<0.01, * * * \mathrm{p}<0.001, \mathrm{~ns}$ : 不显著在服务质量的影响因素两个维度中, 交互媒介的丰 富性对服务质量中的有形性路径系数为 0.242 , 在 0.01 水平 上显著; 交互媒介的丰富性对服务质量中的响应性路径系 数为 0.348 , 在 0.01 水平上显著; 交互媒介的丰富性对服务 质量中的移情性路径系数为 0.441 , 在 0.01 水平上显著。因 此, 研究假设H1a、H1b、H1c成立, 即交互媒介的丰富性 正向促进最后一公里配送服务质量。

自助服务的便利性对服务质量中的有形性路径系数 为 0.470 , 在 0.01 水平上显著; 自助服务的便利性对服务质 量中的响应性路径系数为 0.285 , , 在 0.01 水平上显著; 自 助服务的便利性对服务质量中的移情性路径系数为 0.107 , , 在 0.05 水平上显著; 且这两个维度对服务质量有 形性、响应性、移情性的解释能力分别为 $0.420 、 0.324$ 、 0.265 。假设 $\mathrm{H} 2 \mathrm{a} 、 \mathrm{H} 2 \mathrm{~b} 、 \mathrm{H} 2 \mathrm{c}$ 成立, 即自助服务的便利性 能够显著提升最后一公里配送服务质量。

此外, 中心辐射模式的有形性、响应性和移情性对服 务质量的影响大小分别为 $0.237 、 0.319 、 0.290$, 均在 0.01 水平上显著。假设 $\mathrm{H} 3 \mathrm{a} 、 \mathrm{H} 3 \mathrm{~b} 、 \mathrm{H} 3 \mathrm{c}$ 成立, 即最后一公里配 送的服务质量能够显著提升顾客对服务提供方的信任。

最后, 顾客对服务提供方的信任对满意度的影响大小 为 0.662 , 在 0.01 水平上显著。因此, 假设 $\mathrm{H} 4$ 成立, 即对服
务提供方的信任能够显著提升顾客对最后一公里配送服 务的满意度。

\section{5. 结论}

\section{1. 理论启示}

（1）服务质量的影响因素

一方面, 交互媒介的丰富性越强, 越能提升顾客感受 到的物流配送服务的有形性, 自助服务的便利性越明显, 越能够提升顾客感受到的物流配送服务的有形性, 前者对 有形性影响更加显著, 解释了顾客自助参与业务活动时, 服务设备智能信息化的重要性; 另一方面, 服务质量另外 的两个维度对交互媒介的丰富性、自助服务的便利性的影 响与有形性影响方向一致。且交互媒介的丰富性相对于自 助服务的便利性影响更加显著, 这验证了媒介工具对顾客 感知服务质量的重要性。对于交互媒介的丰富性和自助服 务的便利性与服务质量之间的关系研究中, 前人的探讨较 少, 或者仅仅研究其中的一方面, 没有深层次的把这二者 之间的逻辑关系串联起来。

（2）服务质量对信任的影响

由前文研究内容可以看出, 服务质量与信任二者之间 相辅相成。具体来说, 
由于顾客与服务提供方交互媒介及平台的多样化, 加 上智能化的信息设备, 这两个方面使得顾客认为服务提供 方是真实可靠的, 也相信其满足自己个性化需求的能力, 从而对其提供的配到服务质量体验较好, 最终使得顾客对 服务提供方的硬件设备和服务能力感到信赖, 也就是良好 的服务质量从而保证了顾客对其较高的信任度。在前人的 研究中, 对信任的研究多数基于心理学研究领域, 较少在 经济管理领域探讨, 并且对信任一词的研究停留于表面, 较少从能力、一致性、善意这三个具体组成维度开展进一 步深入的研究。

（3）信任对顾客满意度的影响

顾客对物流配送服务的信任程度越强, 越能够提升顾 客的满意程度, 意味着企业可以重点聚焦顾客对其服务的 信任程度来改善顾客满意度。具体来说, 良好的信任程度 表明顾客十分信赖该服务提供方, 也认为其提供的服务符 合自己的期望。当自己的需求得到满足后, 累积的信任感 会认为自己选择特定的服务方是正确且明智的, 不会为自 己的选择感到后悔或失望, 其结果是使得顾客满意度处于 较高水平。前人研究中, 对于信任的研究大多基于理论的 定性研究, 较少以问卷调查的方式收集全面的数据进行定 量定性相结合的研究。

\section{2. 实践启示}

（1）企业角度

在本文的研究模型基础上, 从企业角度出发, 为了实 现良好的经营效益, 一般需要重点关注顾客满意度这一指 标。具体来说如下所示:

1) 顾客满意度是可以通过访谈或问卷等外在形式了 解到的, 而与其息息相关的服务质量是相对抽象的指标, 因此需要企业管理者重点关注服务质量的影响因素, 如交 互媒介的丰富性、自助服务的便利性等，从根本上保证服 务质量的高水平, 以实现较好的顾客满意度, 为企业市场 经营助力; 另一方面, 企业管理者还应关注服务质量与顾 客满意度之间的中介影响因素, 如信任等, 它是保证顾客 满意度的关键性法宝, 有时其影响力可能胜过服务质量的 影响因素。

2）顾客服务质量与顾客信任存在正向关联，通过对 交互媒介的丰富性、自助服务的便利性的提高，从而提升 用户对服务质量的感知, 进而增加用户的信任程度; 企业 若想提升顾客的信任, 首当其冲应提升顾客对总体服务质 量的感知。

3) 顾客信任程度与顾客满意度存在正相关关系, 意味着企业可以通过提升顾客信任程度来提高顾客满 意度。也就是说, 提升了顾客满意度, 随之而来的即是 用户量和用户黏性的提升, 从而可帮助企业实现更多的 利润。

（2）顾客角度

在实际物流配送活动中, 顾客能直接感受服务提供方 的配送质量, 并且服务质量的影响因素 (交互媒介的丰富 性、自助服务的便利性）感受更为深刻, 例如, 服务设施 是否足够智能信息化、服务提供者是否足够专业化和热情、 是否能借助多种工具及平台完成特定服务环节等, 都是作
为顾客能直接感受的。这些细分维度进一步影响顾客对企 业的信任程度, 如果企业在提供服务过程中, 有意欺瞒顾 客特定信息等不当行为, 会极大的影响顾客对该特定服务 对象的黏性或依赖程度, 并且顾客存在多样化的服务选择 方式, 会使得顾客进行无意识的服务对比后, 对应的顾客 满意度根本无从谈起。

本研究的实践意义主要体现在两个方面：1）在中心 辐射模式的最后一公里背景下, 顾客的直接参与程度和自 主服务选择能力大大增强, 使得服务提供方需要更加关注 服务质量的水平，来在行业竞争中获胜；2）服务提供方 的管理者在企业运作过程中，不仅要关注服务质量本身的 水平, 更要密切关注服务质量的影响因素, 如交互媒介的 丰富性和自助服务的便利性的影响, 以及信任等变量的对 服务质量和顾客满意度的中介作用, 使得服务质量控制效 果更好。

\section{致谢}

本文为国家自然科学基金青年科学基金项目《基于关 系治理视角的商业社群平台使用行为模式研究》 (71502170)的阶段性成果之一。

\section{参考文献}

[1] 张锦,陈义友.物流“最后一公里”问题研究综述 [J].中国流通 经济, 2015, (4): 23-32。

[2] 杨晓红,严中华.基于SERVPERF的高校校园快递“最后一公 里”配送模式研究 [J],2015, 34(7): 16-19。

[3] 张会云, 尚金快.快递业“最后一公里”配送模式分析一以菜 鸟驿站和丰巢为例 $[\mathrm{J}]$. 物流技术, 2015, 22(34): 48-51。

[4] Stanisław Iwan, Kinga Kijewska, Justyna Lemke. Analysis of Parcel Lockers' Efficiency as the Last Mile Delivery Solution - The Results of the Research in Poland [J]. Transportation Research Procedia, 2016, 12: 644-655.

[5] Yael Deutsch, Boaz Golany. A parcel locker network as a solution to the logistics last mile problem [J]. International Journal of Production Research, 2018, 56(1-2): 251-261.

[6] Yulia Vakulenko. Daniel Hellström, Klas Hjort. What's in the parcel locker? Exploring customer value in e-commerce last mile delivery. Journal of Business Research, 2017, in press.

[7] Chris Baumann. Competitiveness vis-à-vis service quality as drivers of customer loyalty mediated by perceptions of regulation and stability in steady and volatile markets $[\mathrm{J}]$. Journal of Retailing and Consumer Services, 2016, 30(15):3-7.

[8] 杨卉. 教师在线实践社区 COP助学服务质量评价体系构建 及应用研究 [J].中国电化教育,2015,34(6):12-42。

[9] 黄永㸓,蒋恵园.基于熵权灰靶理论的港口物流绩效评价 [J]. 工业工程,2015,5(8): 6-11。 
[10] CroninJ J, Taylir S A. Serperf Versus SERVQUAL: Reconciling Performance-Based and Perceptions-Minus-Expectations manangement of Service Quality [J]. Journal of Marketing, 1994, 58(2):125-131.

[11] Goh Mei Ling. Understanding Customer Satisfaction of Internet Banking: A Case Study In Malacca [J]. Procedia Economics and Finance, 2016, 20(5):4-37.

[12] Blaine G. From the general to the specific: How social trust motivates relational trust [J]. Social Science Research, 2016, 12(10):15-15.

[13] Nikolaus Jackob. The tendency to trust as individual predisposition: exploring the associations between interpersonal trust, trust in the media and trust in institutions[J]. The European Journal of Communication Research, 2012, 6(8):9-12.

[14] A Parsu Parasuraman, Valarie A. Zeithaml, Arvind Malhotra. A Multiple-Item Scale for Assessing Elcetronic Service Quality[J]. Journal of Service Research, 2005, 7(3):213-233.

[15] Lalinthorn Marakanon. Perceived quality, perceived risk and customer trust affect customer loyalty of environmentally friendly electronics products $[\mathrm{J}]$. Kasetsart Journal of Social Sciences, 2016, 30(7):14-45.

[16] 张婷. 人机交互界面设计在产品可用性中的应用研究 [J].包 装工程,2014:34-56。

[17] Qian Xu. Interactivity and memory:Information processing of interactive versus non-interactive content $[\mathrm{J}]$. Computers in Human Behavior, 2016, 10(5):18-35.

[18] 间景民.面向顾客的快递业服务质量评价因素体系研究[D]. 大连:大连理工大学,2012。

[19] Jun Yang, Kenneth J, Klassen. How financial markets reflect the benefits of self-service technologies [J]. Journal of Enterprise Information Management, 2008, 21(5):448-467.
[20] 杨涛.基于SEM的图书馆自助服务用户满意和使用研究-以 自助还书系统为例 [J].图书情报工作,2013,20(5):25-31。

[21] 钱慧娜.社区信息服务亭的通用设计研究[D].杭州:浙江工 业大学, 2010。

[22] 张雅洁.兰州市商品住宅小区物业管理务满意度研究[D].兰 州:西北师范大学,2015。

[23] 莫振轩. 我国图书馆自助借阅服务的研究现状与发展趋势 [J].图书馆工作与研究,2013,20(6):22-25。

[24] 罗家德,李智超.乡村社区自组织治理的信任机制初探-以一 个村民经济合作组织为例 [J]. 管理世界.2012,8(10):15-18。

[25] 秦进,赵前前.网络零售服务可靠性五维度对顾客忠诚的影 响-基于顾客满意与顾客信任的视角 $[\mathrm{J}]$.西北工业大学学报 (社会科学版),2014,12(06):20-20。

[26] 罗力.信任和关系承诺对第三方物流整合与绩效的影响 [D]. 广州:华南理工大学, 2010。

[27] 杨浩雄,王雯.第三方物流企业顾客满意度测评体系研究 [J]. 管理评论,2015,33(23):17-49。

[28] Jun He. New Voltage Sag Frequency Evaluation Method Considering the Satisfaction Degree of Sensitive Customer [J]. Advanced Materials Research, 2013, 20(6):37-89.

[29] Parasuraman A, ZeithamlV, Berry L. Reassessment of expectations as a comparison standard in measuring service quality: Implications for further research [J]. Journal of Marketing, 1994, 58(1):111-123.

[30] Walczuch R, Lundgren H. Psychological antecedents of institution-based consumer trust in e-retailing[J]. Information \& Management, 2004, 89(42):159-177.

[31] Farouk S, Nathoo. Bayesian alternatives to null-hypothesis significance testing for repeated-measures designs[J]. Journal of Mathematical Psychology, 2016, 16(4): 69-75. 